\title{
Improvements on the Robustness of Ultrasonic Bearing Measurement Methods
}

\author{
Walter Christian, Schweinzer Herbert, Kaniak Georg \\ Vienna University of Technology, Institute of Electrodynamics, Microwave and Circuit Engineering \\ Gusshausstr. 25/354, A-1040 Vienna
}

\section{Abstract}

Scene analysis has become an important part of ultrasonic research due to its broad applicability in industrial applications, robotics, supervising and other fields. Some reasons why ultrasonic analys is is so attractive for these applications is the inherent reduction of data, high insensitivity to dust or foggy atmosphere and low cost of the sensors. Over time a lot of different methods have evolved which are distinguished by the number of sensors, by targeting 2D or 3D scene analysis and by the type of additional information they employ like distance, object type, polar- and azimuth angle [1-3]. The method presented is special in the sense that it requires only one transmitter and receiver but is still able to perform localization in a 3D environment [4]. This works shows improvements to the existing system.

\section{Introduction}

This work shows further enhancements for a compact sensor for 3D scene analysis [4-7]. The sensor uses a broadband ultrasonic transducer which is required to have a frequency dependent radiation pattern, e.g. one according to the piston membrane model. By restricting ourselves to frequencies below $100 \mathrm{kHz}$ the minimum wavelength in dry air at room temperature $\left(20^{\circ}\right)$ is $3,4 \mathrm{~mm}$. Since the typical roughness of a surface is much smaller than the wavelength we can assume a specular environment and apply the well know laws of reflection. The underlying principle is that by positioning the center of the receiving and transmitting device at approximately the same position it is possible to determine the position of the reflector if the distance is measured and if sound waves emitted into different directions can be distinguished. This is illustrated in Fig. 1 where it can be seen that the ray $d_{1}$, which corresponds to a sound signal emitted at direction $\theta_{1}$ by transmitter $S$, is reflected back to the receiver $R$. The position of the reflection point would therefore be estimated as $P=(r, \theta)=\left(\left(\left|d_{1}\right|+\left|d_{2}\right|\right) / 2, \theta_{1}\right)$. The correct result would be $(d, 0)$ which would be yielded by the algorithm if the transmitter and receiver are exactly at the same position. If only a single microphone is applied this is also the optimum system configuration. It is also well suited to be combined with other methods for localization. For example the multi-receiver system [8] which performs active localization with a single transmitter and multiple receivers by time-of-flight (TOF) measurements is an ideal companion. Together these methods provide increased robustness by adding redundancy and by adding the possibility for detection of multi-path propagation.

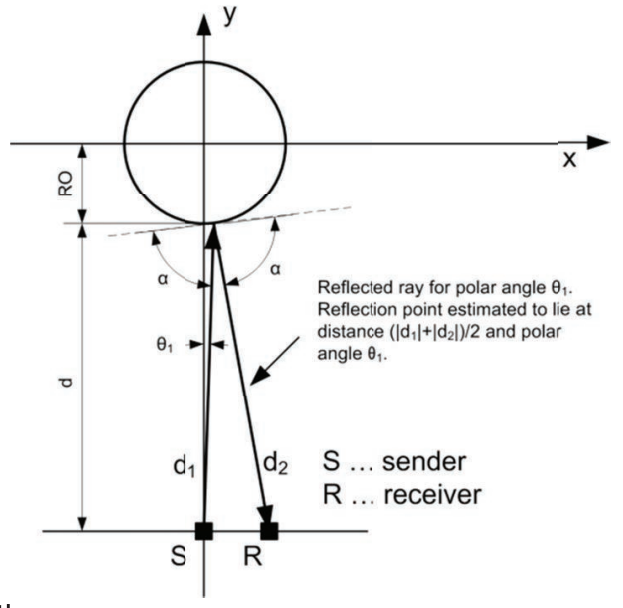

Fig. 1: Principle of localization algorithm

Because the polar (and azimuth) information is encoded within a broadband signal (outside the main lobe) the selection of the transmitting device and the signal waveform has a big impact on system performance. A reasonable choice for a broadband ultrasonic pulse is a linear frequency modulated chirp (LFM) and a piston-membrane type transmitter. The requirement for the frequency modulation originates from the fact that the radiation pattern of the transducer is different for different frequencies as depicted in Fig. 2a. We can see that not only the number of side lobes changes with increasing frequency but also the amplitude. What is not shown in the diagram is that the phase 
between two neighboring side-lobes changes by approx. $180^{\circ}$ assuming a simplified model als in [9]. The exact form of the radiation pattern is a function of the diameter of the piston membrane and the signal frequency [10]. Fig. $2 \mathrm{~b}$ shows two different rays $\operatorname{Ray}_{1}=\left(r, \theta_{1}, \varphi_{1}\right) \operatorname{Ray}_{2}=\left(r, \theta_{2}, \varphi_{2}\right)$ emitted into different directions. We can see that the signals differ in amplitude and in their zero crossings. The values used for simulation are a piston type membrane model with a diameter of $38 \mathrm{~mm}$ and a LFM-chirp from $35 \mathrm{kHz}$ to $75 \mathrm{kHz}$ with a length of $750 \mu \mathrm{s}$. These values are matched to the SensComp 600 environmental grade transducer which is also used in the experimental setup.
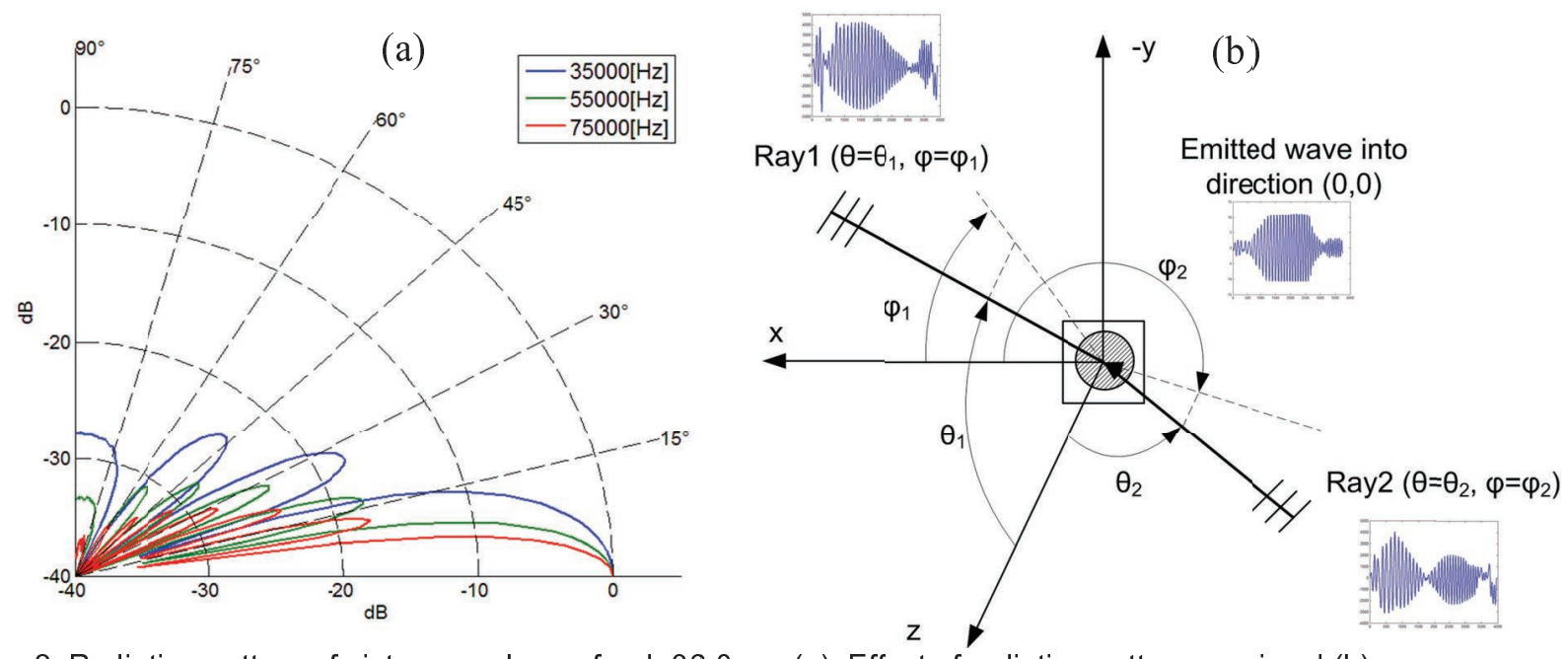

Fig. 2: Radiation pattern of piston membrane for $d=36.0 \mathrm{~mm}(a)$, Effect of radiation pattern on signal (b)

In addition the choice of a LFM chirp is common in radar applications because in combination with matched filtering it allows good range resolution [11]. The choice of a piston-membrane type transducer is a trade of between costs, price and availability.

\section{Algorithm}

Having outlined the basic principles behind the algorithm we will now present the algorithm in full detail. We start by defining the used terms and symbols: The coordinate system shall be a spherical coordinate system with its origin at the center of the transmitter as show in Fig. 2b. Coordinates are expressed in terms of distance, polar angle and azimuth angle and written in vector notation as $(r, \theta, \varphi)$. Vectors are indicated by an upper-case first letter. Time dependent signals are written as all lower-case letters. System constants are written as all upper-case letters.

Def. 1: Digital correlation based on polarity conversion: Write $x *_{b} y$ for the binary correlation between two time discrete signals and $x *_{b n} y$ for the normalized binary correlation.

The usage of the binary correlation is beneficial because the signal amplitude of the reflection is dependent on the object (surface roughness, type, etc.), the distance and other factors. For more information on binary correlation the reader is referred to [12].

Def. 2: Driving signal: The (electrical) driving signal for the transmitter is fully determined by the type of modulation, its bandwidth and amplitude levels. It is assumed to stay constant.

Def. 3: Measured signal: A signal MeasSig captured by an ultrasonic microphone. The time index 0 is always assumed to be aligned with the transmission of the driving signal for proper TOF measurements.

Def. 4: Received signal: A received signal RecvSig is assumed to belong to a single echo of a driving signal. If derived from a measured signal it is assumed to be zeroed outside the window of the echo signal to keep TOF information.

Def. 5: Reference signals: A reference signal RefSig is a received signal or mathematically derived signal from the driving signal. Let $\left\{\operatorname{RefSig}_{1}, \ldots, \operatorname{RefSig}_{M}\right\}$ be the set of all $M$ reference signals.

Def. 6: Vector definition signal: A vector definition signal VectSig is a received signal with a known direction vector $(1, \theta, \varphi)$.

Def. 7: Reference vector: A reference vector RefVec is derived from the set of reference signals and a received or mathematically derived vector definition signal VectSig with the components given by eq. (1).

$$
\operatorname{RefVec}_{i}=\max \left(\operatorname{VectSig} *_{b n} \operatorname{RefSig}_{i}\right)
$$


Fig. 3 shows an example for reference vectors for different directions built from $M=20$ reference signals.

Def. 8: Comparison vector: Built from a received signal RecvSig and the set of reference signals RefSignals $=$ $\left\{\operatorname{RefSig}_{1}, \ldots, \operatorname{RefSig}_{M}\right\}$ with the components given by eq. (2).

$$
\operatorname{CompVec}_{i}=\max \left(\operatorname{RecvSig}_{b n} \operatorname{RefSig}_{i}\right)
$$
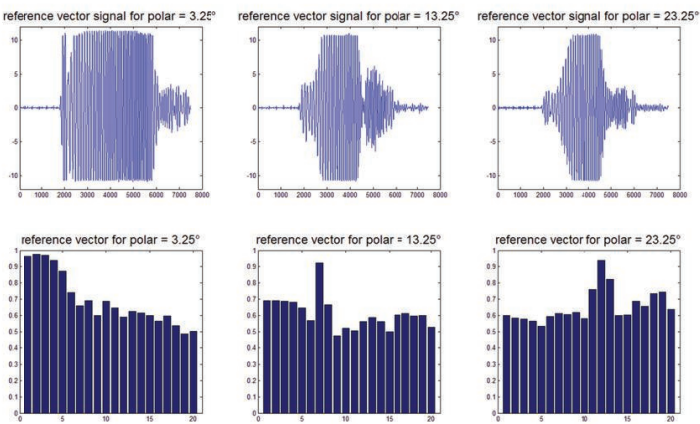

Fig. 3: Example for reference vectors built from 20 reference signals for different polar anglles.

Def. 9: Least square error between vectors: The least square error between two vectors elements of size $M$ is given by

$$
L S Q(V e c 1, V e c 2)=\sum_{m=0}^{M}\left(V e c 1_{m}-V e c 2_{m}\right)^{2}
$$

Fig. 4 shows the algorithm as a block diagram. A normalized binary cross correlation between the received 1-bit quantized waveform RecvSig and the reference signals is computed yielding $M$ new signals $\operatorname{RecvSig} *_{b n} \operatorname{RefSig}{ }_{i} 1 \leq$ $i \leq M$. The time of flight is obtained from the signal with the highest correlation peak since we cannot assume a-priori knowledge of the angle of reflection.

$$
\begin{gathered}
\text { TOF }=\operatorname{argmax}\left(\operatorname{RecvSig}^{*} \operatorname{RefSig}_{i}\right) \text { and } \\
\forall i \neq j \max \left(\operatorname{RecvSig}_{*} * \operatorname{RefSig}{ }_{i}\right) \geq \max \left(\operatorname{RecvSig}_{\operatorname{RefSig}}\right) 1 \leq i \leq M
\end{gathered}
$$

In the next step the comparison vector is obtained by applying Def. 8. This vector is then compared to any of the reference vectors and the best matching reference vector is chosen. The comparison is performed by a least-squares compare according to Def. 9. The estimated location for the reflection point is then given by the vector definition signal and the TOF information.

Fig. 4: TOF and polar/azimuth detection algorithm

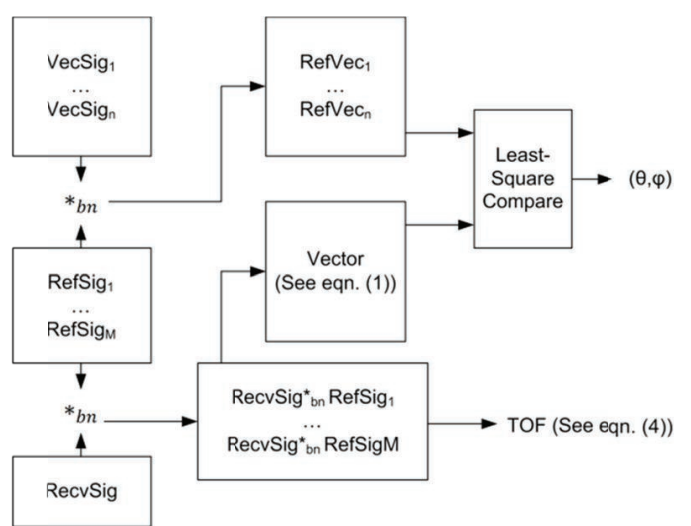

\section{Improvements}

Fig. 5a shows a typical input signal received from the test scenario shown in Fig. 5b. What can be seen is that in a typical environment there are a lot of different echoes. While the algorithm above works fine in case of a single echo it contains restrictions making it hard to use in a general setup. Because of the maximum operation required by Def. 7 a window would be required for the input signal to correctly build the vector. The size of such a window needs to be at least the size of the chirp including some margin which is a serious restriction if echoes are close to each other. The required windows are also indicated in Fig. 5a as a dashed rectangle around the signals. In addition the starting position of the window would either have to be dependent on the signal amplitude or would have to be based on the TOF estimates from the reference signals. In the first case a signal like the one at $1,17 \mathrm{~m}$ would be hard to detect with a windowing algorithm based on amplitude information because of the low SNR.

To solve this problem we have enhanced the algorithm such that it does no longer require the use of the maximum operation for a measurement but only for building the reference vectors. The last requirement is easy to achieve 
because the reference vectors can be measured in a well-defined environment or can be created synthetically if a model of the transmitter is available. The basic idea behind the modification is that we do not only store the amplitude of the correlation peaks but also their relative time displacement. This is shown in Fig. 6a and Fig. 6b where two examples for the binary correlation between a reference vector signal and the reference signals are shown.

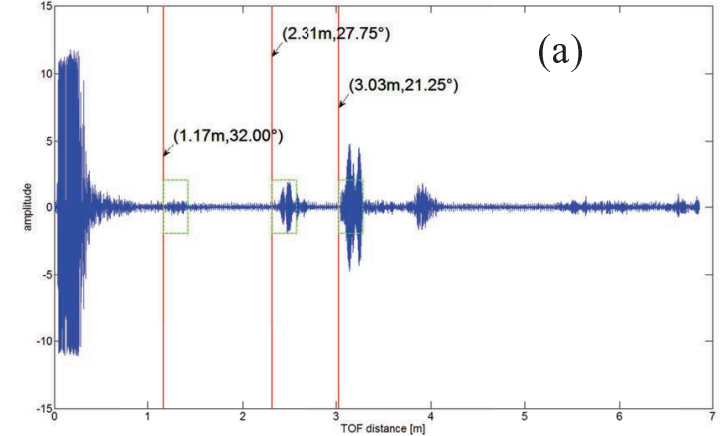

Fig. 5: Typical received input signal (a) left and test setup

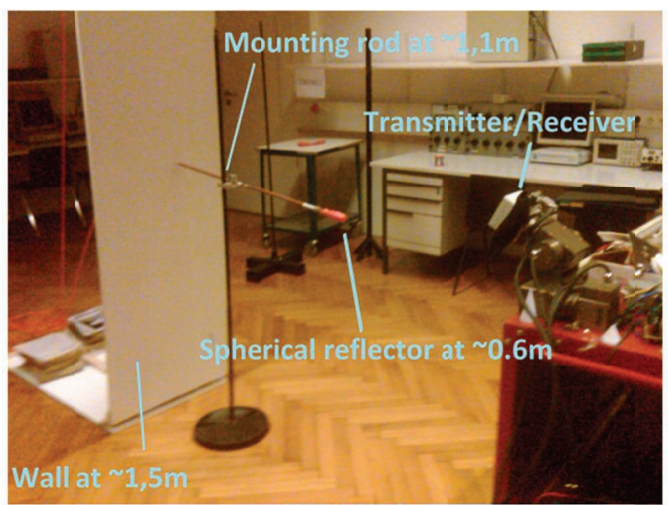

(b)

(b) on the right side.

Fig. $6 \mathrm{c}$ shows see the amplitude reference vector and Fig. $6 \mathrm{~d}$ the time reference vector. The time vector was normalized to the peak position of Fig. $6 \mathrm{~b}$ but this does not matter because only the relative displacements will be used onwards.
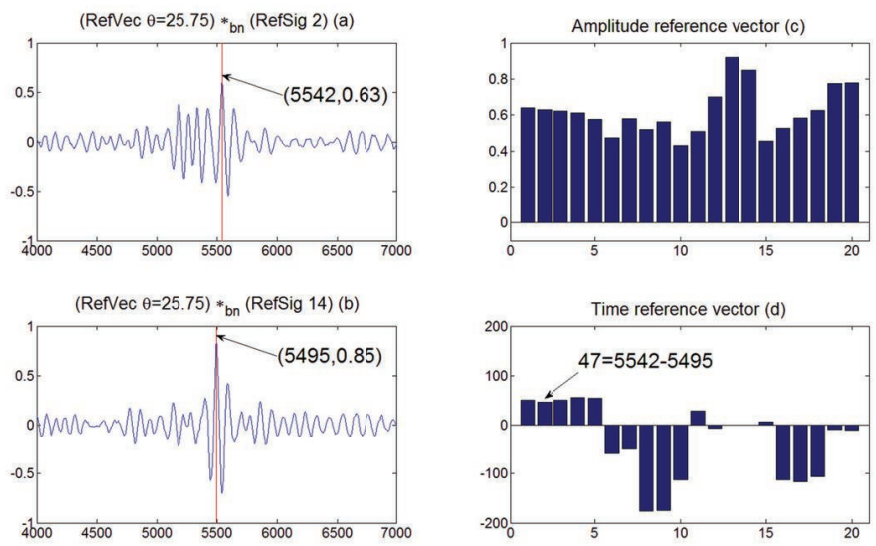

Fig. 6: Correlation between reference signals and reference vectors signals (a, b), amplitude reference vector (c) and time reference vector $(\mathrm{d})$

Having the relative displacements between each vector element it now suffices to find a starting point for the search of the corresponding reference vector. This is show in Fig. 7 a-3 where the correlation results between the measured signal and reference signal 12 has been plotted over the full time range. Peaks above a defined threshold are used as a starting point and are marked with an asterisk $*$. The process of finding the correct reference vector also needs to be changed. Each tested reference vector is aligned to the currently used reference signal (12 in the example shown in Fig. 7 a-3). The comparison vector is then picked from the correlation results lby only using the time differences and no longer by a maximum operation. This is shown in Fig. 7b for the comparison vector for $\theta=$ $28,5^{\circ}$ built from the amplitudes marked with a red star indicated in Fig. 7 a1-a4. Similarly the comparison vector in Fig. 7c for $\theta=13,75^{\circ}$ has been built from the amplitudes marked with a cyan plus in Fig. 7 a1-a4. Fig. 7d and Fig. 7e show the difference between the (stored) reference vector and the comparison vector. In this case the least-square algorithm would choose $\theta=28,5^{\circ}$ because the difference shown in Fig. $7 \mathrm{~d}$ is smaller than in Fig. 7e. We will now give the new definitions for the computation of the reference vectors and the comparison vectors.

Def. 10: Reference vector pair: An additional vector is appended to the reference vector delivering a vector pair RefVec and RefVecTime. Both vectors are derived from a set of reference signals RefSignals $=\left\{\right.$ RefSig $\left._{1}, \ldots, \operatorname{RefSig}_{M}\right\}$ and a measured or mathematically derived vector definition signal VectSig .

$$
\begin{aligned}
& \text { RefVec }_{i}=\max \left(\operatorname{VectSig}_{{ }_{b n}} \operatorname{RefSig}_{i}\right) \\
& \text { RefVecTime }_{i}=\operatorname{argmax}\left(\text { VectSig }_{b n} \text { RefSig }_{j}\right)
\end{aligned}
$$

Modified Def. 8*: Comparison vector: Let MeasSig $*_{b n}$ RefSig $_{i}$ be the input streams for $1 \leq i \leq M$. Let $t_{j}$ be a peak found in the input stream MeasSig ${ }_{b n} \operatorname{RefSig}_{j}$. The comparison vector for a reference vector pair RefVec and RefVecTime is then computed as following.

$$
\begin{aligned}
& \text { RefVecTimeAligned }_{i}=\text { RefVecTime }_{i}-\text { RefVecTime }_{j}(\text { Zero align }) \\
& \text { CompVec }_{i}=\left(\text { MeasSig }_{\text {bn }} \text { RefSig }_{i}\right)\left[t_{j}+\text { RefVecTimeAligned }_{i}\right]
\end{aligned}
$$


Because the search can be conducted multiple times for the same echoes an additional output aggregation step is required. This aggregation step creates groups for approximately the same distances and then builds the median of
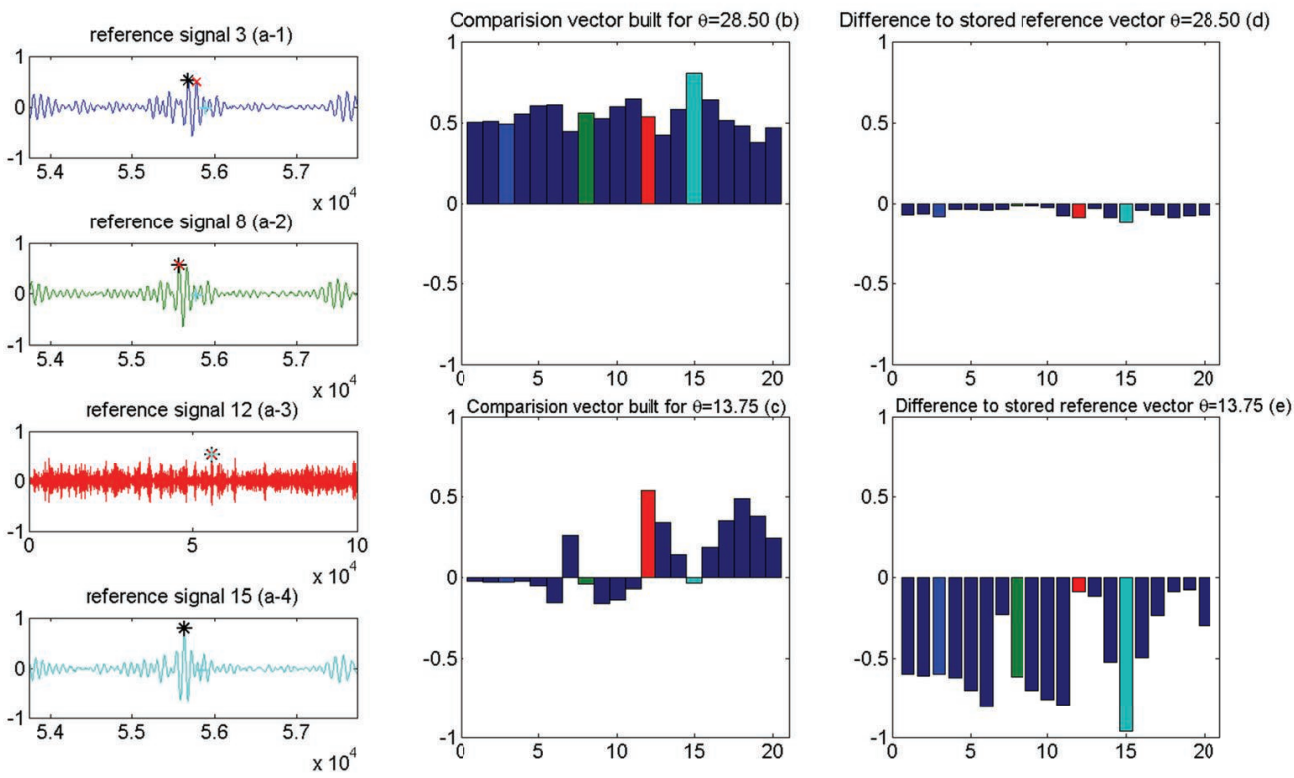

Fig. 7: Correlation of received signal with reference signals (a1-a4), comparison vector for $\theta=28,5^{\circ}$ (b), comparison vector for $\theta=13,75^{\circ}(\mathrm{c})$, difference to reference vector for $\theta=28,5^{\circ}(\mathrm{d})$, difference to reference vector for $\theta=13,75^{\circ}$ (e).

the distances and the polar- and azimuth angle results. Using the median is an easy way to deal with single outliers. For the example above the algorithm yields the following results

\begin{tabular}{|l|l|l|l|l|}
\hline Distance & Polar Angle & $\begin{array}{l}\text { Size of } \\
\text { Group }\end{array}$ & $\begin{array}{l}\text { Normalized median absolute } \\
\text { deviation for polar angle }\end{array}$ & $\begin{array}{l}\text { Normalized median absolute } \\
\text { deviation for distance }\end{array}$ \\
\hline $0.59 \mathrm{~m}$ & $28^{\circ}$ & 1 & 0 & 0 \\
\hline $1.163 \mathrm{~m}$ & $35^{\circ}$ & 11 & $0.13^{\circ}$ & $670 \mathrm{um}$ \\
\hline $1 . .521 \mathrm{~m}$ & $25^{\circ}$ & 25 & $1.4257^{\circ}$ & $440 \mathrm{um}$ \\
\hline
\end{tabular}

In Fig. 8 we show the results from an actual measurement were the transducer was rotated around its $x$-axis. Since the objects are fixed the reflection point stays the same in world coordinates but of course in the reference frame the movement of the transducer can be seen. The detection ratio of the mounting rod is not good because the reflection of the floor becomes a problem as well as the sharp corners on the rod due to the mounting of the shaft.
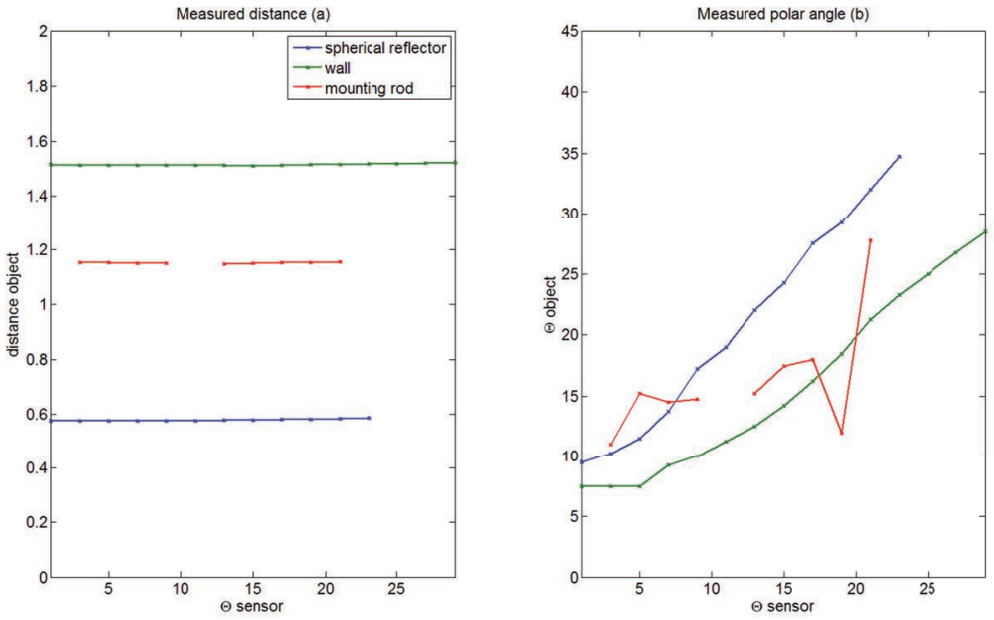

Fig. 8: Measurement with transducer rotation around the $\mathrm{x}$-axis $=\theta$ sensor

In addition we have added an automatic scaling of reference vectors which improves performance in case the SNR of the received signal is low. The reason is that a low SNR reduces the correlation amplitude of the input signal according to [10]. The definition is given below and the improvements are shown in Fig 9. We can see that without the scaling (method standard) performance drops rapidly as shown in the red curve in the cumulative distribution 
function (CDF). With scaling (method standard2) all errors are below $5^{\circ}$ even with an increase of $30 \mathrm{~dB}$ in noise corresponding to an SNR of 0 for higher polar angles.

Modified Def. 10*: Least square error between vectors: The least square errors between two vectors elements of size $M$ is given by

$$
\operatorname{ELSQ}(\operatorname{Vec} 1, \operatorname{Vec} 2)=\sum_{m=0}^{M}\left(\operatorname{Vec} 1_{m}-c \cdot V e c 2_{m}\right)^{2} \text { with } c=\sum_{m=0}^{M} V e c 1_{m} \cdot V e c 2_{m} / \sum_{m=0}^{M} V e c 1_{m}{ }^{2}
$$

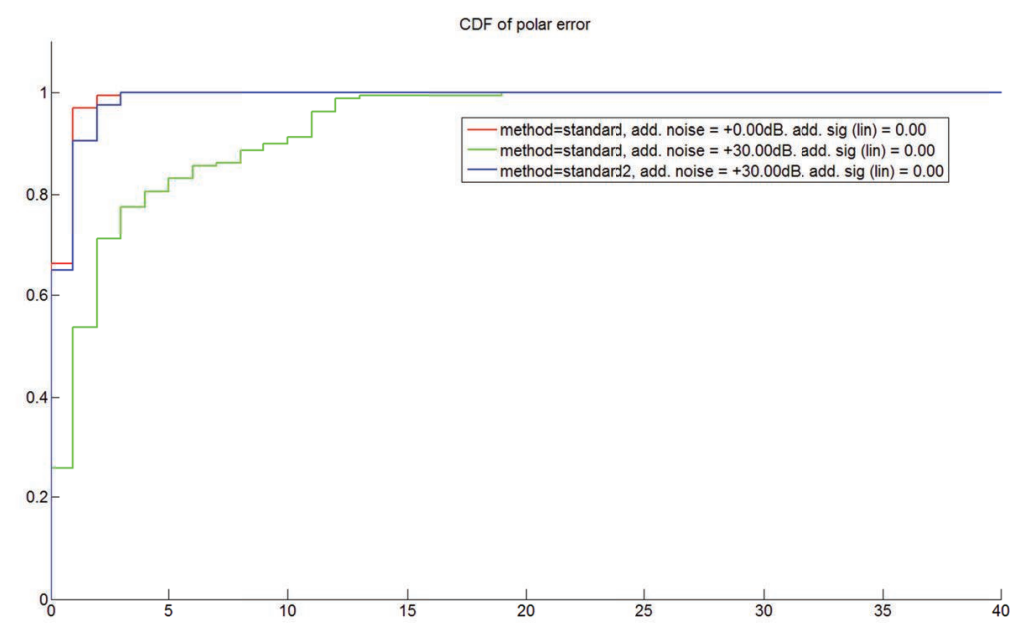

Fig 9: Noise improvements with scaling

\section{Conclusion}

We have presented our suggested approach for 3D localization using a system consisting of only a single transmitter and receiver. Furthermore we have presented a modification to the algorithm which makes it more easy to use in practice by avoiding the need for a window function. For this modified algorithm we have added some practical results showing its suitability for real world problems. We have finished our work by showing how robustness can be further enhanced with a scaling of the reference vectors during the comparison process.

\section{References}

[1] Akbarally H., Kleeman L., A sonar sensor for accurate 3D target localization and classification, 1995 IEEE International Conference on Robotics and Automation, pp. 3003-3008 vol.3, 21-27 May 1995

[2] Peremans H., Walker A., Hallam J.C.T., 3D object localization with a binaural sonarhead, inspirations from biology, 1998 IEEE International Conference on Robotics and Automation, pp. 2795-2800 vol.4, 16-20 May 1998

[3] Barshan B., Location and curvature estimation of spherical targets using multiple sonar time-of-flight measurements, IEEE Transactions on Instrumentation and Measurement, pp.1212-1223, Dec 1999

[4] Kaniak G., Schweinzer H., Advanced Ultrasound Object Detection in Air by Intensive Use of Sidelobes of Transducer Radiation Pattern, IEEE Sensors Conference, Sensors 2008, Lecce, Italy, 26-29 October 2008

[5] Kaniak G., Schweinzer H., Comparison of Two Methods for Determining the Position of Reflection Points Using Ultrasound Echolocation in a Specular Environment, Proceedings of the Sensor+Test Conferrence 2009, ISBN: 9783-9810993-4-8, Nürnberg, Germany, 26.05.2009 - 28.05.2009

[6] Kaniak G., Schweinzer H., Principles for echo position determination using airborne ultrasound, International Symposium on Measurement Technology and Intelligent Instruments, Saint Petersburg, Russia; 29.06.2009 02.07.2009

[7] Kaniak G., Schweinzer H., Magerl G., Precise Echolocation and Characterization of Objects in Air by Broadband Ultrasonic Transducers, tm - Technisches Messen 4/2008, ISSN 0171-8096, Oldenburg Verlag, 2008

[8] Kaniak G., Schweinzer, H. , 3D Airborne Ultrasound Sensor for High-Precision Location Data Estimation and Conjunction, Instrumentation and Measurement Technology Conference Proceedings 2008, IMTC 2008 IEEE , pp.842-847, 12.05.2008 - 15.05.2009.

[9] Zollner M.: Elektroakustik, Springer, Berlin, $3^{\text {rd }}$ edition, ISBN 3540646655, 2003

[10] Elmer H., Schweinzer Herbert: Ultrasonic Distance Measurement System with a Well Defined and Adjustable Detection Area, Proceedings IEEE Sensors, vol 1, p. 437, Vienna, Oct. 2004

[11] Cook C.E., Pulse Compression-Key to More Efficient Radar Transmission, Proceedings of the IRE, vol.48, no.3, pp. 310-316, March 1960.

[12] Elmer H, Schweinzer H.: Performance considerations of ultrasonic distance measurement with well defined properties, Journal of Physics: Conference Series, vol 13, p. 133, 2005 